\title{
Investigation of amyloid deposition in uterine leiomyoma patients
}

\author{
Jinping Liu', Fei Zhai ${ }^{1}$, Peng Ge ${ }^{2}$, Jinhai $\mathrm{Lu}^{2}$, Yi Qin ${ }^{2}$, Xuguo Sun ${ }^{2 *}$ \\ ${ }^{1}$ Department of Neurology, Tianjin Medical University Hospital, Tianjin, China \\ ${ }^{2}$ Department of Medical Laboratory College, Tianjin Medical University, Tianjin, China; \\ *Corresponding Author: sunxuguo@tijmu.edu.cn
}

Received 21 June 2012; revised 17 July 2012; accepted 26 July 2012

\section{ABSTRACT}

Objects: To investigate the pathogenesis of amyloid presented in uterine leiomyoma. Methods: 36 uterine leiomyoma patients were recruited and divided into two groups according to Congo red staining results. 6 cases are Congo red staining-positive, and 30 cases Congo red staining-negative which represented amyloid positive and amyloid negative respectively. All patients' serum total protein (TP), albumin (Alb) and prealbumin (PA) levels were measured as well as blood hemoglobin $(\mathrm{Hb})$, cell counts of white blood cell (WBC), neutrophils (NEU) and lymphocyte (LYM). Glycogen in tissue was compared between amyloid accumulated and amyloid negative sections with periodic acid schiff staining (PAS) in leiomyoma patients. Results: All of blood $\mathrm{Hb}$ concentration, WBC, NEU and LYM have not been found significant differences between two groups. Also no obvious infiltration of inflammatory cells was observed in tissue with amyloid deposition in uterine leiomyoma patients. And levels of TP, Alb and prealbumin have not been found significant differences between two groups. The amyloid was negative in leiomyoma entity cells range by Congo red staining, while small blood vessels in myoma tissues were positively detected with high rate. Amyloid was found in normal tissue around myoma as well as in blood vessel of pseudo-capsule. Increased PAS-positive material induced by leiomyoma was not correlated with amyloid deposition. Conclusions: Metabolic changes in the setting of functional alterations of cell in local microenvironment with uterine leiomyoma, may be related to the amyloid deposition.

Keywords: Amyloid; Uterine Leiomyoma;
Pathogenesis

\section{INTRODUCTION}

Uterine leiomyoma is the most common benign tumor of female genital mutilation, mainly found in $30-50$ year-old women, of which morbidity reaches $20 \%-30 \%$ [1]. Large uterine leiomyoma can cause pain, constipation, frequency of urination and increased menstrual pain and irregularity.

The pathogenesis of uterine leiomyoma is still unclear, but evidence suggests that growth of cells is tied to estrogen e.g. overexpression of mRNA of progesterone receptor in the surface of uterine leiomyoma revealed an activated phenotype of progestational proliferation, plausibly related to the growth of uterine leiomyoma [2]. In vitro, uterine leiomyoma cells have a significantly higher response to estrogen than do matched myometrial culture [3]. Rogers, R., et al. [4] found that extracellular matrix (ECM) including collagen and glycosaminoglycan were highly expressed in leiomyoma which was associated with increased stiffness of leiomyoma. What's more, ECM alteration was accompanied with hyaluronic acid and sulfuric acid decreased but keratan sulfate and dermatan sulfate significantly increased [5].

Amyloidosis represents a spectrum of diseases that result from deposition of amyloid in extracellular matrix, leading to disruption of normal function and a broad but nonspecific clinical manifestations [6]. Systemic amyloid can be found in the human heart, kidney, intestine, nerve, gland, etc. Up to 25 different types of amyloid precursor proteins have been described. Recently, amyloid was reported in many benign tumors and chronic diseases [7, 8]. In 2000, Wu lin [9] found amyloid in uterine leiomyoma. As a kind of benign tumors, researchers believed that leiomyoma growth was relative to estrogen metabolism and as year well on proliferation of leiomyoma cells would be inhibited so it was generally accepted that treatment of uterine leiomyoma was conservative. However, damage from amyloid in human body become se- 
verer as amyloid deposition increased. Elucidation of mechanism of amyloid deposited in uterine leiomyoma patients will be helpful to establish a scientific principle of treatment and preventive measures of uterine leiomyoma.

By studying blood cells, serum biochemical substances and analysis of the biochemical composition changes in interstitial tissue from leiomyoma patients with positive and negative amyloid deposition, we want to investigate the mechanism of amyloid formation in uterine leiomyoma.

\section{MATERIALS AND METHODS}

\subsection{Patients}

According to the clinical and pathological diagnostic criteria for diagnosis of intramural uterine leiomyoma, 36 patients were enrolled with the exception of having liver, heart or kidney disease.

\subsection{Detection of Blood Cells and Hemoglobin}

Venous blood was sampled two days before surgery, anticoagulated with heparin. Blood cells as well as hemoglobin $(\mathrm{Hb})$ were analyzed within 30 minutes using Sysmex F-820 automatic blood analyzers and reagents.

\subsection{Determination of Serum Biochemical Index}

Total protein, albumin and prealbumin (PA) of each patient were determined by TOSHIBA TBA-120FRautomatic biochemical analyzer (Toshiba Corporation, Japan), reagents supplied by Shanghai Rongsheng biological reagents Ltd.

\subsection{Congo Red Staining}

Uterus specimens after surgery were fixed by formalin-PBS within half an hour, embedded with paraffin and typically sectioned. Sections were deparaffinized, hydrated to water and then placed directly into $1 \%$ Congo red working solution at room temperature for 1 hour. After incubation, the sections were rinsed in distilled water for 1 minute. Sections were immersed in Harris hematoxylin for 10 minutes then washed with distilled water for 10 minutes and bluing treated with $0.2 \%$ ammonia 20\% ethanol solution. Finally, dehydrated in alcohol, cleared and coverslipped.

\subsection{Periodic Acid Schiff Staining}

Sections deparaffinized and hydrated to water, then immersed in $0.5 \%$ periodic acid for 5 minutes and rinsed in distilled water. Schiff reagent was reacted with rinsed section at room temperature for 20 minutes and then washed in running tap water for 10 minutes. Later, counterstained in hematoxylin for 3 minutes. Finally, dehydrated in graded alcohols, cleared in xylene and coverslipped.

\section{STATISTICAL ANALYSES}

The statistical analyses were performed by the SPSS for windows version 11.0 software. The differences between groups were analyzed with independent samples T-test. P-value < 0.05 was considered significant. All measurement data were expressed as mean \pm standard deviation.

\section{RESULTS}

\subsection{Comparison of Blood Cells and $\mathrm{Hb}$ between Two Groups}

Indicators reflecting the anemia and infection through blood routine examination between leiomyoma patients with positive and negative amyloid deposition: there was no significant difference of $\mathrm{Hb}$ between two groups $(\mathrm{P}=$ $0.505,>0.05$ ); no significant differences of white blood cells and absolute neutrophil count was seen between two groups, neither absolute lymphocyte count was (Table 1).

\subsection{Serum Proteins}

Serums from patients with leiomyoma Congo red staining positive and negative were collected and total protein, albumin and prealbumin were determined. There were no significant differences of the three proteins between two groups (Table 2).

Table 1. Comparison of cell counts and Hb between two groups.

\begin{tabular}{ccc}
\hline Index & $\begin{array}{c}\text { Congo red-positive } \\
(\mathrm{n}=6)\end{array}$ & $\begin{array}{c}\text { Congo red-negative } \\
(\mathrm{n}=30)\end{array}$ \\
\hline $\mathrm{Hb}(\mathrm{g} / \mathrm{L})$ & $120.5 \pm 16.8$ & $115.3 \pm 19.5$ \\
$\begin{array}{c}\text { White blood cell } \\
\left(\times 10^{9} / \mathrm{L}\right)\end{array}$ & $6.6 \pm 1.4$ & $5.8 \pm 1.6$ \\
$\begin{array}{c}\text { Absolute neutrophil } \\
\left(\times 10^{9} / \mathrm{L}\right)\end{array}$ & $3.7 \pm 1.9$ & $3.2 \pm 1.3$ \\
$\begin{array}{c}\text { Absolute lymphocyte } \\
\left(\times 10^{9} / \mathrm{L}\right)\end{array}$ & $2.3 \pm 0.4$ & $1.9 \pm 0.6$ \\
\hline
\end{tabular}

Table 2. Comparison of serum proteins between two groups.

\begin{tabular}{cccc}
\hline Group & $\mathrm{TP}(\mathrm{g} / \mathrm{L})$ & $\mathrm{Alb}(\mathrm{g} / \mathrm{L})$ & $\mathrm{PA}(\mathrm{g} / \mathrm{L})$ \\
\hline $\begin{array}{c}\text { Congo red-positive } \\
(\mathrm{n}=6)\end{array}$ & $67.25 \pm 4.58$ & $39.77 \pm 2.86$ & $0.26 \pm 0.043$ \\
$\begin{array}{c}\text { Congo red-negative } \\
(\mathrm{n}=30)\end{array}$ & $68.63 \pm 5.93$ & $41.62 \pm 2.90$ & $0.28 \pm 0.057$ \\
\hline
\end{tabular}

Abbreviations: TP: Total protein; Alb: Albumin; PA: Prealbumin; CR-positive: Congo red staining positive; CR-negative: Congo red staining negative. 


\subsection{Inflammation in Amyloid Deposited Tissue}

Surgical specimens diagnosed as leiomyoma by pathologist were stained with H\&E and Congo red. Under light microscopy inflammatory cell infiltration was rarely observed around CR positive smooth muscle fiber or in CR positive arterioles wall and inflammatory cell number was not significantly different in these areas compared with CR negative tissue (Figure 1).

\subsection{Amyloid Distribution in Leiomyoma Tissue}

It was found that amyloid was deposited mainly in tumor vessel and vascular wall of fibroid pseudo-capsule while leiomyoma entity was amyloid negative (Table 3).

\subsection{PAS-Positive Material and the Relationship with CR}

Sections of 36 cases were all stained with H\&E, CR and PAS and observed comparison of the scope of PASpositive material distribution with the amyloid deposition within area from pseudo-capsule and vessel wall of leiomyoma. PAS-positive materials were highly expressed in smooth muscle fibers and vascular intimal stromal side, however, overlapping staining of PAS and CR was not in complete accord (Figure 2). Histomorphologically, there was no correlation between the amount of PASpositive materials in interstitial tissue and amyloid deposition in uterine leiomyoma.

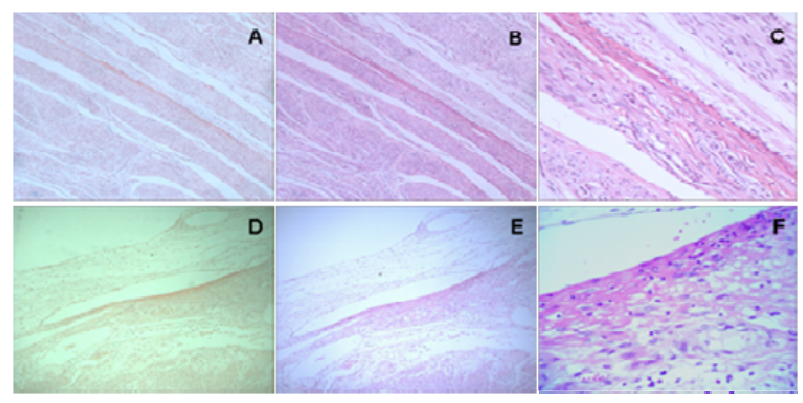

Figure 1. H\&E staining in the initial specimen shows no significant inflammatory cell infiltration in CR-positive and CRnegative tissue. CR staining: (A) and (D); H\&E staining: (B), (C), (E) and (F). (A)-(E) $10 \times 10$; (C), (F) $40 \times 10$.

Table 3. Distribution of leiomyoma amyloid.

\begin{tabular}{cc}
\hline Distribution & Percent (\%) \\
\hline Tumor interstitium & $0 \%$ \\
Tumor vessel & $50 \%$ \\
Tumor periphery & $16.7 \%$ \\
Pseudo-capsule vessel & $83.3 \%$ \\
Pseudo-capsule fiber & $33.3 \%$ \\
\hline
\end{tabular}

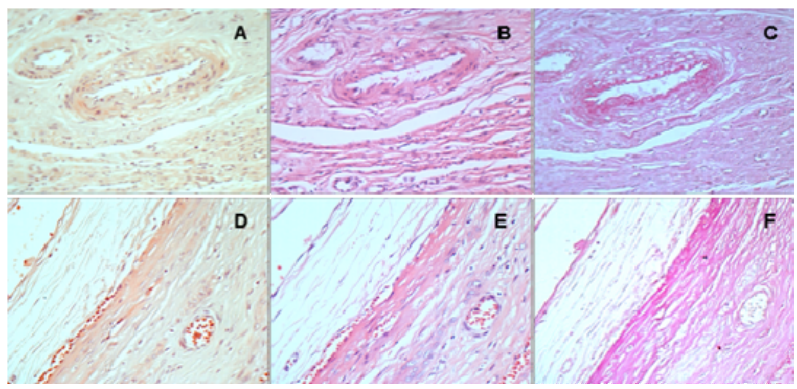

Figure 2. Scope of CR staining and PAS staining in leiomyoma tissue with amyloid. CR staining: (A) and (D); H\&E staining: (B) and (E); PAS staining: (C) and (F).

\section{DISCUSSION}

H\&E staining revealed there was no significant inflammatory cell invasion in amyloid deposition site of uterine leiomyoma and indicators reflecting the infection were not significantly different between two groups through patients' physicochemical examination. There was no significant difference of serum protein content between leiomyoma amyloid positive and negative patients. It was found that amyloid deposition was negative for fibroblast interstitium of uterine fibroid entity while pseudo-capsule and blood vessels around the fibroid amyloid deposition was positive. Amyloid deposition was almost found in the peripheral and vascular tissue of tumor entities, all in extracellular matrix.

Serum TP, Alb and serum PA levels which reflecting the nutritional status were not shown significant differences between amyloid positive and negative groups (Table 1). To investigate the impact of recent infection to amyloid, blood cell counts and Hb level were detected and no significant differences were seen between two groups. Histological sections of leiomyoma specimen were stained with H\&E and Congo red to elucidate the relationship between inflammation and amyloid and in amyloid deposited area, there was no increased inflammatory cells, which suggests that infection was not directly associated with amyloid formation.

In leiomyoma, myofibroblast was proliferated and ECM was overexpressed. Amyloid deposited in tissue looked as block mass. It may be the key to analyze the interstitial changes for elucidation of amyloid formation in leiomyoma. Specimens of fibroids stained with H\&E, CR and PAS shown that all the muscle fiber interstitiums within fibroid entities were negative for amyloid deposition while fibroid capsule and vascular amyloid deposition positive which suggest that blood-derived ingredients and/or interstitium affected by metabolism of leiomyoma, may lead to conformational change of amyloid precursor protein and gradual amyloid deposition. On the other hand, histomorphologically, there was no correlation between the PAS-positive material distribution in 
interstitial tissue and amyloid deposition in uterine leiomyoma, indicating that increased secretion of fibroids was not straight accompanied with amyloid formation. Fibroids, with complete pseudo-capsule, vigorous tumor cells metabolism and overproduction of free radical caused increased peroxidation of surrounding tissue. Importantly, peroxide markers were recognized in amyliod deposition by immunohistochemical and biochemical techniques [10]. It was proved that amyloidogenic protein stereostructure would become more unstable under protein chemical modification [11-13]. Inference from these results and our findings support that metabolic changes in the setting of functional alterations in local microenvironment with uterine leiomyoma, may be related to the amyloid deposition.

It was found that all the muscle fiber interstitiums within fibroid entities amyloid deposition was not observed while fibroid capsule and vascular wall were amyloid deposition positive. In fact, almost all the amyloid was deposited in the tumor entities surrounding tissues and vascular structures. We concluded that local tissue metabolism changes resulted from fibroid cell functional alterations, may be related to the amyloid deposition. However, amyloidogenic mechanism and impact of amyloid in leiomyoma should be further investigated.

\section{ACKNOWLEDGEMENTS}

The authors thank Prof. Ando Yukio for their helpful providing the Congo red solution and the control tissue specimens for the study.

\section{REFERENCES}

[1] Lurie, S., Piper, I., Woliovitch, I. and Glezerman, M. (2005) Age-related prevalence of sonographicaly confirmed uterinene myomas. Journal of Obstetrics and Gynaecology, 25, 42-44. doi:10.1080/01443610400024583

[2] Fujimoto, J., Hirose, R., Ichigo, S., Sakaguchi, H., Li, Y. and Tamaya, T. (1998) Expression of progesterone receptor form A and B mRNAs in uterine leiomyoma. Tumour Biology, 19, 126-131. doi:10.1159/000029983

[3] Andersen, J., DyReyes, V.M., Barbieri, R.L., Coachman, D.M. and Miksicek, R.J. (1995) Leiomyoma primary cultures have elevated transcriptional response to estrogen compared with autologous myometrial cultures. Journal of the Society for Gynecologic Investigation, 2, 542551. doi:10.1016/1071-5576(94)00053-4

[4] Rogers, R., Norian, J., Malik, M., Christman, G., AbuAsab, M., Chen, F., et al. (2008) Mechanical homeostasis is altered in uterine leiomyoma. American Journal of $\mathrm{Ob}$ stetrics and Gynecology, 198, 474.e1-11.

[5] Mitropoulou, T.N., Theocharis, A.D., Stagiannis, K.D. and Karamanos, N.K. (2001) Identification, quantification and fine structural characterization of glycosaminoglycans from uterine leiomyoma and normal myometrium. Biochimie, 83, 529-536. doi:10.1016/S0300-9084(01)01281-0

[6] Sun, X.G., Hu, K.S., Song, L. and Feng, Q. (2009) Diagnosis of amyloidosis. Journal of Chinese Modern Medicine, 6, 292-295.

[7] Zhang, Q.H., Ren, Y.P. and Zhang, Q. (2004) 1 case of pituitary prolactin cell adenoma with amyloid-containing substances. International Journal of Clinical and Experimental Pathology, 20, 511-512.

[8] Bestard, M.O., Poveda, M.R., Ibernon, V.M., Carrera, P.M. and Grinyó, B.J.M. (2008) Systemic AA amyloidosis induced by benign neoplasms. Nefrologia, 28, 93-98.

[9] Wu, L., Yi, G.W. and Lei, J.X. (2000) 22 cases of pregnancy with uterine fibroids. Journal of Fourth Military Medical University, 21, 310.

[10] Ando, Y., Nyhlin, N., Suhr, O., Holmgren, G., Uchida, K. and Yamashita, T. (1997) Oxidative stress is found in amyloid deposits in systemic amyloidosis. Biochemical Biophysical Research Communications, 232, 497-502. doi:10.1006/bbrc.1996.5997

[11] Liu, J.P., Zheng, F., Liu, X.C., Li, X., Song, J.Y. and Sun, X.G. (2008) Chemical modification of transthyretin in familial amyloidotic polyneuropathy. Journal of Fourth Military Medical University, 29, 883-885.

[12] Sun, X.G., Liu, J.P., Wang, Q.Y. and Ni, E. (2007) Peroxidative modification and amyloidogenesis. Chinese Journal of Coal Industry Medicine, 10, 1345-1347.

[13] Connors, L.H., Jiang, Y., Budnik, M., Théberge, R., Prokaeva, T., Bodi, K.L., et al. (2007) Heterogeneity in primary structure, post-translational modifications, and germline gene usage of nine full-length amyloidogenic kappal immunoglobulin light chains. Biochemistry, 46, 1425914271. doi:10.1021/bi7013773 